OPEN ACCESS

Edited by:

Songyan Liao,

The University of Hong Kong,

Hong Kong

Reviewed by:

Yali Jia,

Academy of Military Medical Sciences

(AMMS), China

Zhang Kaiji,

Chengdu Integrated TCM and

Western Medical Hospital, China

*Correspondence:

Xin Du

miyadu@hotmail.com

†These authors share first authorship

Specialty section:

This article was submitted to

Stem Cell Research,

a section of the journal

Frontiers in Cell and Developmental

Biology

Received: 14 October 2020 Accepted: 31 December 2020

Published: 11 February 2021

Citation:

Chen X, Li N, Weng J and Du X (2021)

Senescent Mesenchymal Stem Cells in Myelodysplastic Syndrome:

Functional Alterations, Molecular Mechanisms, and Therapeutic

Strategies.

Front. Cell Dev. Biol. 8:617466. doi: 10.3389/fcell.2020.617466

\section{Senescent Mesenchymal Stem Cells in Myelodysplastic Syndrome: Functional Alterations, Molecular Mechanisms, and Therapeutic Strategies}

\author{
Xiaofang Chen ${ }^{1 \dagger}$, Ningyu $\mathrm{Li}^{1,2 \dagger}$, Jianyu Weng ${ }^{1,2}$ and Xin $\mathrm{Du}^{1,2 *}$ \\ ${ }^{1}$ Department of Hematology, Guangdong Provincial People's Hospital, Guangdong Academy of Medical Sciences, \\ Guangzhou, China, ${ }^{2}$ School of Medicine, South China University of Technology, Guangzhou, China
}

Myelodysplastic syndrome (MDS) is a group of clonal hematopoietic disorders related to hematopoietic stem and progenitor cell dysfunction. However, therapies that are currently used to target hematopoietic stem cells are not effective. These therapies are able to slow the evolution toward acute myeloid leukemia but cannot eradicate the disease. Mesenchymal stem cells (MSCs) have been identified as one of the main cellular components of the bone marrow microenvironment, which plays an indispensable role in normal hematopoiesis. When functional and regenerative capacities of aging MSCs are diminished, some enter replicative senescence, which promotes inflammation and disease progression. Recent studies that investigated the contribution of bone marrow microenvironment and MSCs to the initiation and progression of the disease have offered new insights into the MDS. This review presents the latest updates on the role of MSCs in the MDS and discusses potential targets for the treatment of MDS.

Keywords: mesenchymal stem cells, senescence, myelodysplastic syndrome, bone marrow microenvironment, treatment

\section{INTRODUCTION}

Myelodysplastic syndromes (MDS) lead to a clonal disease of the hematopoietic system, characterized by ineffective hematopoiesis and a high risk of transforming into acute myeloid leukemia (AML).

In most studies, MDS is considered a hematopoietic cell disorder in which disease initiation and progression are exclusively driven by hematopoietic cell-intrinsic genetic events. During the past 10 years, a large number of studies have been conducted on the genetic and molecular aspects of cloned cells in MDS, and more than 40 gene mutations associated with the prognosis of patients, as well as treatment targets, have been revealed. However, these mutations are not specific and have been found in normal people as well as patients with idiopathic cytopenia of undetermined significance (ICUS), where a certain proportion of the latter population eventually develops MDS (Glenthøj et al., 2016). Therefore, the mechanisms underlying MDS initiation and progression cannot be fully attributed to genetic and molecular changes alone. Several earlier observations have challenged this reductionist view, and a large number of studies have shown that MDS is associated with an abnormal bone marrow (BM) microenvironment. 
The post-birth hematopoietic microenvironment is mainly located in the BM and comprises interstitial cells, helper cells, and sympathetic nerve cells. Bone abnormalities such as "adynamic" bone, characterized by reduced osteoblast numbers, decreased mineral apposition rates, and osteoporosis, have been noted in MDS patients in comparison with agematched controls (Mellibovsky et al., 1996; Weidner et al., 2017). Mesenchymal stem cells (MSCs), which are a source of stromal cells in the hematopoietic environment, have subsequently been identified as key components of this disrupted architecture. Ex vivo-expanded MSCs display altered differentiation characteristics, transcriptional abnormalities, and a reduced ability to support hematopoietic stem/progenitor cells (HSPCs) in MDS, suggesting that MSCs may play a potential role in BM failure seen in MDS (Raaijmakers, 2012; $\mathrm{Li}$ and Calvi, 2017). MSCs play a crucial role in the BM microenvironment (Kfoury and Scadden, 2015; Pleyer et al., 2016). These cells display a potential for self-renewal and multidirectional differentiation and may differentiate into a variety of mesenchymal cells, such as osteoblasts, adipocytes, and chondrocytes. Precise regulation of hematopoietic stem cells (HSCs) maintains hematopoiesis for life. In addition, MSCs also display immunoregulatory functions, by maintaining the stability of the BM immune microenvironment and reducing the damage caused to HSCs by stress stimuli. Animal studies have shown that genetic abnormalities in MSCs are sufficient to induce MDS formation. Dysfunctional MSCs also play an important role in the progression of MDS and its transformation to AML.

At present, the understanding of genetics and gene expression characteristics associated with MDS-derived MSCs remains limited, and experimental results pertaining to the morphology, proliferation, differentiation, and hematopoiesis show inconsistencies. However, the association between functional changes in aging MDS-MSCs and disease progression, as well as the relevance of such changes to prognostic evaluation and treatment, is increasingly attracting the attention of researchers. Thus, a better understanding of the role played by MSCs in the pathogenesis of MDS may help strengthen knowledge regarding the complexity of MDS pathogenesis and help determine new treatment options.

This review describes current target-HSC treatments and elaborates on the role of the BM microenvironment in MDS. The importance of MSC senescence and phenotypic characteristics, as potential targets for MDS treatment, is discussed. This review may help improve existing knowledge regarding the initiation and progression of MDS and enable new targets for the treatment of MDS to be determined.

\section{CURRENT STATUS OF MDS TREATMENT}

Treatment of MDS, which is based on the WHO Prognostic Scoring System (WPSS) and the International Prognostic Scoring System (IPSS and IPSS-R) for risk stratification, involves individualized treatment measures (Ferrer et al., 2013). For the MDS treatment algorithm, all patients should receive appropriate supportive treatment. Since then, the MDS expert group proposed to initially divide patients with clinically significant cytopenias into two main risk groups: (A) lower-risk patients [including IPSS low, intermediate-1; IPSS-R very low, low, and intermediate ( $\leq 3.5)$; and WHO-based Prognostic Scoring System for risk stratification (WPSS) very low, low, and intermediate] and (B) higher-risk patients [including IPSS intermediate- 2 and high; IPSS-R intermediate (>3.5), high, and very high; and WPSS high and very high]. In addition, intermediate-risk patients with disease that does not respond to therapy for lower-risk disease would be eligible to receive therapy for higher-risk MDS. In addition, intermediate-risk patients who do not respond to treatment of lower-risk diseases are eligible for treatment of higher-risk MDS.

Patients with low-risk MDS are provided with supportive treatment using cytokines and immunomodulatory drugs, such as lenalidomide. Patients with $\operatorname{del}(5 \mathrm{q})\left(5 \mathrm{q}^{-}\right)$chromosomal abnormalities with or without one other cytogenetic abnormality, except those involving chromosome 7 and symptomatic anemia should be treated with lenalidomide. Studies have shown the relative safety of lenalidomide in these patients and the improved quality-of-life (QOL) outcomes in randomized clinical trials (Oliva et al., 2013; Revicki et al., 2013). Patients without the $5 \mathrm{q}^{-}$ abnormality, with or without one other cytogenetic abnormality and accompanied by symptomatic anemia, are categorized by serum erythropoietin (EPO) levels. These patients should receive erythropoietin, G-CSF, or iron repletion treatment (HellströmLindberg, 1995; Negrin et al., 1996; Hellström-Lindberg et al., 1997; Casadevall et al., 2004; Spiriti et al., 2005) (Figure 1). Patients without symptomatic anemia, with increased BM blasts or any other clinically relevant cytopenias, should be considered for treatment with decitabine, azacytidine, immunosuppressive therapy, or a clinical trial (Jabbour et al., 2017).

Patients with high-risk MDS are administered demethylating drugs, such as decitabine and azacytidine, or allogeneic HSC transplantation, which is considered the only possible cure for MDS (Figure 1). However, due to limitations associated with the application of transplantation technology to MDS patients, most patients are found to be unsuitable for this treatment program (Alessandrino et al., 2008; Hicks et al., 2013; Oliva et al., 2013; Revicki et al., 2013). In therapeutic trials, the panel, using the standardized International Working Group (IWG) response criteria for evaluating studies, found it important to stipulate that all MDS patients should be given relevant supportive care (Cheson et al., 2000, 2006; Hicks et al., 2013; Pfeilstöcker et al., 2016). The latest developments pertaining to MDS treatment during the past 10 years indicate that lenalidomide and decitabine may improve the hematology of low-risk and high-risk patients, respectively, and that azacytidine is the only drug that may prolong the overall survival of high-risk MDS patients (Silverman et al., 2011). However, lenalidomide does not delay the progression of patients to high-risk MDS and subsequent conversion to AML. Failure of MDS treatments to achieve a breakthrough may be due to the fact that genetic and molecular abnormalities in the cloned cells associated with MDS do not clearly relate to events leading to the onset of MDS or to disease progression.

Therefore, new drugs developed to counter different molecular mechanisms related to MDS-cloned cells have been largely ineffective. These results indicated that other mechanisms may be involved in the initiation and progression of MDS. 


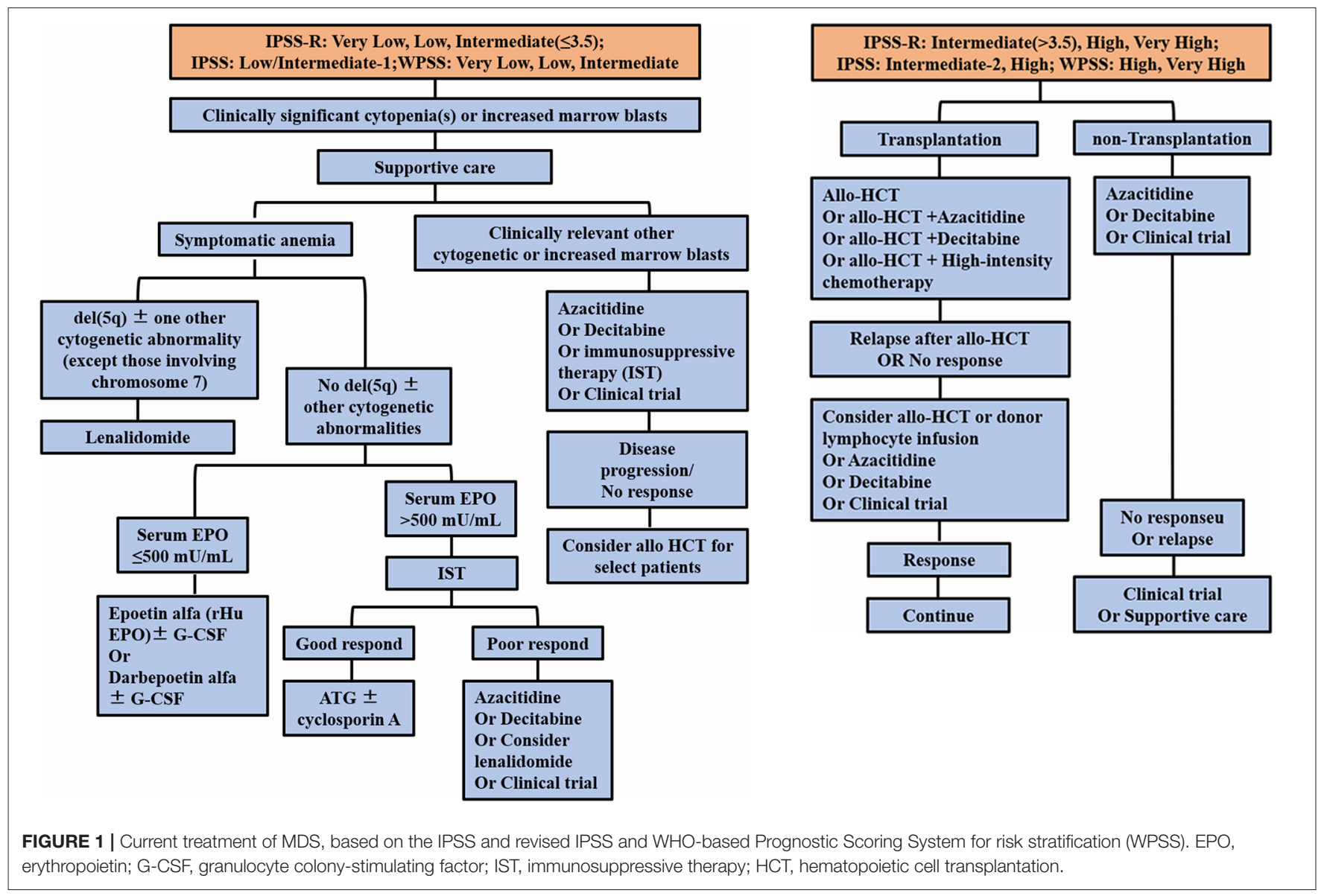

Mouse MDS models, such as Dicer1-knockout and NHD13 mice, exhibited MSC dysfunction and MDS-like morbidity hematopoiesis, which play an important role in the progression of MDS disease (Lin et al., 2005; Balderman et al., 2016). These results indicated that the dysfunctional MSCs derived from MDS are related to the pathogenesis of MDS.

\section{THE ROLE OF BM MICROENVIRONMENT IN MDS}

It has long been considered that hematopoietic cell disorders, which are solely driven by genetic events, are capable of inducing the initiation and progression of MDS. However, several previous studies have suggested that the BM microenvironment also contributes to MDS pathogenesis (Verstovsek et al., 2002; Iwata et al., 2007; Ferrer et al., 2013; Geyh et al., 2013). The BM microenvironment plays an essential role in the maintenance and development of HSPCs. It regulates quiescence, self-renewal, proliferation, and differentiation of stem cells (Adams and Scadden, 2006; Li and Li, 2006; Moore and Lemischka, 2006). The cells in the BM microenvironment are dynamic in nature and may shape the environment to favor an abnormal population of niche residents via certain mechanisms. Colmone et al. reported that the interaction between normal HSPCs and the $\mathrm{BM}$ was influenced by a leukemia cell line (Colmone et al.,
2008). More definitively, genetic changes in hematopoietic cells cause secondary changes in the cells constituting the BM microenvironment, which supports abnormal hematopoietic populations (Schepers et al., 2013). Bone abnormalities in MDS patients such as "adynamic" bone, displayed decreased osteoblast numbers and osteoporosis, compared to age-matched controls (Mellibovsky et al., 1996; Weidner et al., 2017).

Prior to hematopoietic cell transplantation, chemotherapy and radiation therapy were used to exert direct effects on HSCs and the microenvironment and markedly changed the normal supportive BM environment (Barcellos-Hoff et al., 2005; Wright, 2005). Mice that were irradiated following transplantation of growth factor-dependent syngeneic mouse BM cells developed leukemia at a faster rate and in higher numbers compared with non-irradiated recipients (Dührsen and Metcalf, 1990). Thus, in allogeneic patient BM environments, normal transplant-donor cells are transformed into hematopoietic neoplastic cells, demonstrating that the BM environment may contribute to the initiation of MDS (Flynn and Kaufman, 2007). Although infrequent, it appears that interaction between the BM microenvironment and genetically aberrant HSPCs may induce MDS pathogenesis. Thus, during the last decade, the BM microenvironment has been shown to exhibit a potentially permissive or causative role, challenging the belief that hematopoietic cell disorder is solely an element of the initiation and progression of MDS. 


\section{MSC SENESCENCE AND PHENOTYPIC CHARACTERISTICS IN MDS}

MSCs, which display pluripotent and undifferentiated capacities, are key components of the BM microenvironment (Sacchetti et al., 2007). In recent years, evidence has increasingly demonstrated that MSCs in MDS are intrinsically pathological and that senescence is increased by a continuous decline in proliferation (Ferrer et al., 2013; Geyh et al., 2013). Starting from birth and proceeding into adulthood, the composition of BM stromal cells changes over time. MSCs and HSCs/HSPCs that colonize adult $\mathrm{BM}$ during development undergo changes in composition over time. During later stages of fetal development, skeletal stem cells (SSCs), which are characterized by the expression of Osx (encoding osterix), Sox9 (encoding SRY-box 9), and Col2a1 (encoding collagen type II $\alpha 1$ chain), produce bone and BM stromal cells, which persist postnatally (Maes et al., 2010; Mizoguchi et al., 2014; Ono et al., 2014b). However, as depicted in Figure 2, SSCs are not labeled by these markers, while stromal cells are depleted during adulthood (Maes et al., 2010; Park et al., 2012; Mizoguchi et al., 2014; Ono et al., 2014b). Neural crest-derived cells make a short-term contribution to BM stromal cells and the activity of colony-forming unit fibroblasts (CFUFs) during the early postnatal period, but these cells decrease in adulthood too and are replaced by nonneural crest-derived stromal cells (Takashima et al., 2007; Komada et al., 2012; Isern et al., 2014). Consistent with this, Nes-CreER ${ }^{+}$(Nes encodes nestin) stromal cells promote bone formation in early postnatal BM but exert little or no effect on osteogenesis or CFU-F activity in adult BM (Figure 2) (Ono et al., 2014a; Zhou et al., 2014). By contrast, leptin receptor-expressing cells appear in the BM postnatally and exert an effect on the stromal cell population or osteogenesis. As numbers increase, these become the main source of adipocytes and osteoblasts in adults over time (Figure 2) (Méndez-Ferrer et al., 2010; Zhou et al., 2014).

Cellular senescence, a complex process that is usually accompanied by functional changes, is a special state of cell cycle arrest in proliferating cells under the stimulation of stress factors, wherein cells undergo a series of changes in morphology, proliferation, differentiation, secretion, and other functional abnormalities (Pleyer et al., 2016). Changes in morphology intuitively reflect the phenomenon of senescence in MDSMSCs. MSCs in the donor group are usually slender fibers or fusiform, whereas the volume of MDS-MSC is obviously increased, showing a flat irregular polygon. A cytoskeletal morphology study showed that the morphological changes in MDS-MSCs were related to increased, as well as disorderly Factin distribution. The expression level of $\beta$-galactosidase is significantly increased in senescent cells. Staining experiments indicated that $\beta$-galactosidase in MDS-MSCs was significantly increased, directly illustrating the aging of MSCs in MDS (Ferrer et al., 2013; Geyh et al., 2013; Fei et al., 2014; Zhao et al., 2015). Senescence of MDS-MSCs also manifests as a decline in proliferation ability. Parameters such as CFU-F, cumulative number of passages, and doubling time were significantly worse in the MDS-MSC group compared to those of control MSCs. The proliferation ability of MDS-MSCs was significantly reduced. Such a decrease in MSC proliferation capacity may be related to increases in cell telomerase length, chromosome methylation status, abnormal proliferation regulation signal pathway, cell cycle arrest, and other factors (Pavlaki et al., 2014; Falconi et al., 2016).

Decreased differentiation in aging MDS-MSCs both in vivo and in vitro was associated with the senescence and aging of MSCs (Stenderup et al., 2003; Bonab et al., 2006; Zhou et al., 2008). Alizarin Red chemical staining showed that the osteogenic differentiation potential of MDS-MSCs decreased significantly during the in vitro induction of differentiating MSCs into osteoblasts (Ferrer et al., 2013; Geyh et al., 2013; Fei et al., 2014; Zhao et al., 2015). The expression levels of genes involved in osteogenic differentiation accurately indicated the differences between the osteogenic differentiation potentials of cells. The transcription factors, RUNX2 and Osterix, involved in regulating early osteogenic differentiation and genes encoding osteocalcin, serve as mature osteogenic markers in MDS-MSCs. The expression levels of these were significantly lower than those of the control group, indicating that the osteogenic differentiation potential of MSCs in aging MDS had decreased (Ferrer et al., 2013; Geyh et al., 2013; Fei et al., 2014; Zhao et al., 2015).

Senescent cells still show metabolic activity, but many cytokines associated with senescence in MDS-MSCs, such as transforming growth factor $\beta 1$ (TGF $\beta 1$ ), HGF, Jagged1, angiopoietin-1, osteopontin, CXCL-12, IL-6, TGF- $\beta$, SCF, and VEGF, are expressed abnormally. Jagged 1 is a ligand involved in the Notch signaling pathway, the activation of which plays an important regulatory role in the differentiation of HSCs and the formation of myeloid tumors (Geyh et al., 2013; Pavlaki et al., 2014). The expression of jagged-1 in MDS-MSCs was significantly increased, and 38\% of MDS tumor cells showed excessive activation of the Notch signaling pathway (Geyh et al., 2013; Pavlaki et al., 2014). S100A9 promotes cellular senescence of BM stromal cells via TLR4, NLRP3 inflammasome formation, and IL$1 \beta$ secretion (Shi et al., 2019). Some reports have indicated that MSCs exhibited insufficient hematopoietic support capability in MDS compared with donor cells (Zhao et al., 2012; Ferrer et al., 2013). Finally, MSCs from MDS were more prone to cellular senescence than donor MSCs, where senescent MDSMSCs exhibited reduced differentiation potential and stem cell support capacity.

\section{CHANGES IN THE FUNCTION OF AGING MDS-MSC TO SUPPORT HEMATOPOIESIS}

As described above, in vivo mouse experiments have demonstrated that abnormal MSCs may affect the initiation and progression of MDS. Knocking out Dicer1, which encodes endonuclease III of miRNA in mouse MSCs, caused MDS-like morbid hematopoiesis in the $\mathrm{BM}$, transforming a portion of the mouse population into AML (Kfoury and Scadden, 2015). Several mouse models have been developed to mimic human MDS, of which the mouse NUP98-HOXD13 (NHD13) transgenic model, wherein MSCs and osteoblast dysfunction play an important role in MDS progression, may be the most accurate (Lin et al., 2005; Balderman et al., 2016). Following 


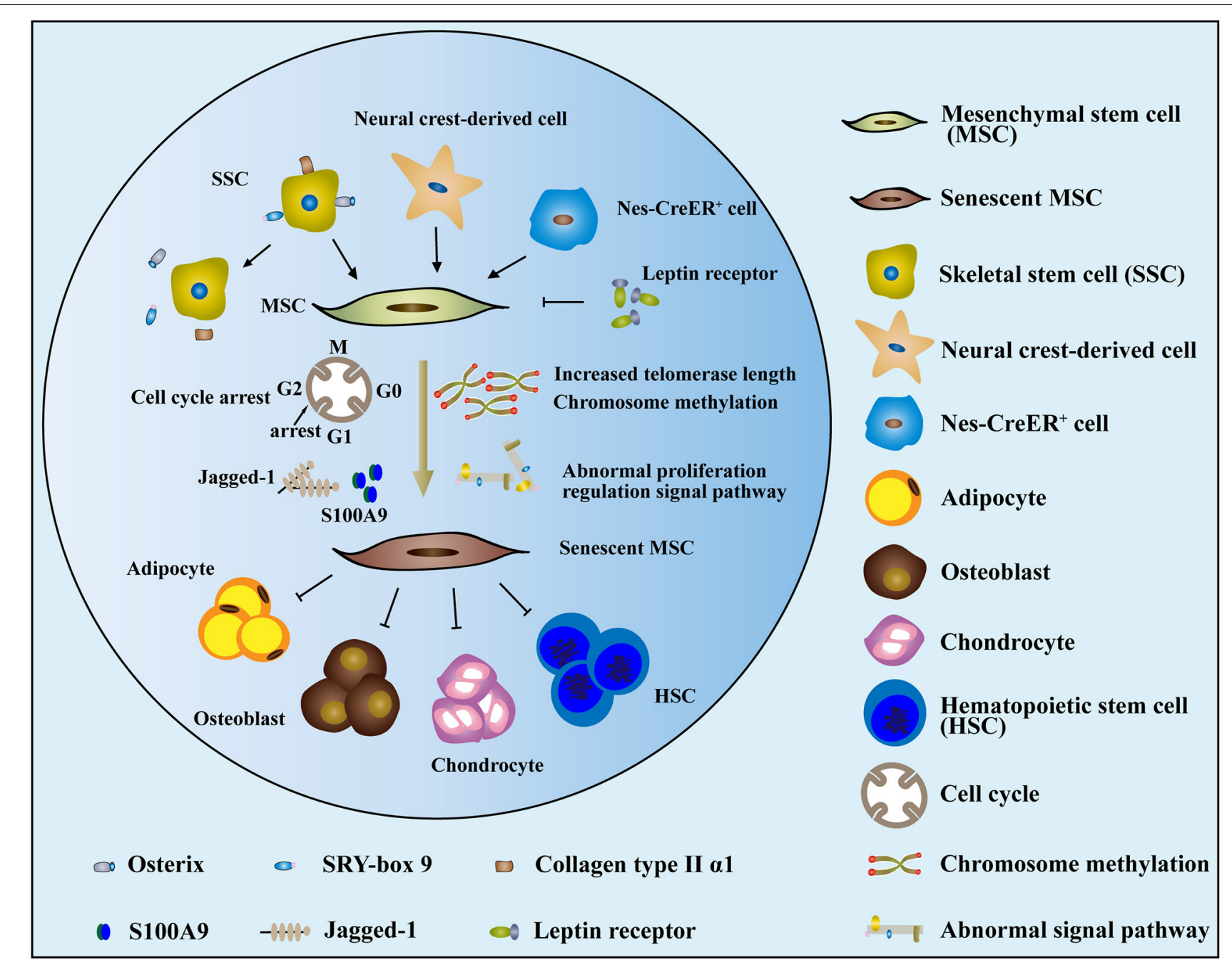

FIGURE 2 | The mechanism and dysfunction of MSC senescence in MDS. (1) Cells that contributed to MSCs, such as SSCs, neural crest-derived cells, and nes-CreER+ stromal cells, were reduced or depleted in MDS. In contrast, leptin receptors, which are detrimental to the function of MSCs, increased over time. (2) The senescence of MDS-MSCs may be related to increases in cell telomerase length, chromosome methylation status, abnormal proliferation regulation signal pathway, cell cycle arrest, and other factors. (3) The senescent MDS-MSCs exhibited reduced differentiation potential and stem cell support capacity.

transplantation of NHD13 mouse hematopoietic cells into both NHD13 and WT mice, the rates of AML transformation and mortality in NHD13 mice were found to be significantly higher than those in WT mice. By contrast, hematopoiesis of NHD13 mice transplanted with BM from WT mice showed a myeloid bias, while the hematopoietic function of WT mice was impaired due to dysfunctional MSCs (Balderman et al., 2016).

Abnormal functioning of proliferation, differentiation, and secretion of aging MDS-MSCs leads to changes in the regulation of hematopoietic function. In an in vitro coculture experiment using MDS-MSCs and donor HSCs, the proportion of hematopoietic cells in the G1 phase decreased while the proportion in the G0 phase increased, in a manner which was significantly different from that in normal MSCs $(P=0.0076)$ (Geyh et al., 2013). MDS-MSCs showed a significantly altered cell cycle status and displayed a shift toward increased apoptosis compared to control MSCs. These changes may contribute to the pathogenesis of MDS (Abbas et al., 2019).

High levels of hyaluronan (HA) were detected in the BM sera of higher-risk MDS patients in comparison with those of donor controls. High levels of HA in BM serum, which enhances osteogenic differentiation of MSCs, were associated with adverse clinical outcomes and significantly shorter median survival in MDS (Fei et al., 2018).

Defective proliferation was observed in pediatric MDSderived MSCs. Pediatric MDS-derived MSCs were more prone to cellular senescence than healthy controls and showed a decrease in the S phase (Liu et al., 2015). Iron overload (IO) reportedly promotes mitochondrial fragmentation and enhances autophagy in MSCs of MDS patients by activating the AMPK/MFF/Drp1 pathway (Zheng et al., 2018). These results indicated that dysfunctional MSCs derived from MDS were associated with MDS-associated pathogenesis. 


\section{MSCs AS A POTENTIAL TARGET FOR MDS TREATMENT}

The functions of MDS-MSCs not only play an important role in the initiation and progression of the disease but also provide a protective microenvironment for tumor cells, which is a poor prognostic factor of MDS. Improving MDS-MSC function as an option for MDS treatment has gradually advanced from its theoretical basis to the research level. Co-cultivation experiments using HSCs in vitro have shown that MDS-MSCs inhibited erythroid hematopoiesis and promoted myeloid cell production, significantly restoring the contention that MDS-MSCs support erythroid hematopoiesis following lenalidomide treatment (Geyh et al., 2013). Demethylation drugs may simultaneously affect the functions of cloned cells and MSCs in MDS. Following transplantation of NHD13 mouse hematopoietic cells into normal mouse BM, the rates of transformation to acute leukemia and death were significantly reduced, while hemoglobin and white blood cell indicators in peripheral blood were enhanced (Balderman et al., 2016). These data indicated that improving MSC function may delay disease progression and enhance cytopenia of MDS.

However, the genetic and molecular changes occurring in MDS cloned cells do not fully clarify the initiation of MDS and disease progression. This may be an important factor affecting the treatment of hematopoietic cells, where most patients will eventually show a declining treatment response. Thus, treatment of MDS cloned cells alone may not produce a cure. However, intervention involving clonal cells and the BM microenvironment may lead to a new treatment strategy for MDS.

MDS-MSCs showed increased production of proinflammatory cytokines. Treatment with 5-azacytidine significantly decreased IL-6 levels in MDS-MSCs in vitro, compared to the IL-6 levels in MSCs from the donor. As MSCs produce much more inflammatory cytokines involved in MDS pathogenesis, these may represent a potential therapeutic target. Moreover, 5-azacytidine may exert a stromal effect, thereby modulating the immune response in MDS (Boada et al., 2020).

Following the administration of $\alpha$-lipoic acid (ALA), the levels of reactive oxygen species (ROS) in MSCs were gradually decreased, intracellular iron content was reduced, and the potential and integrity of the mitochondrial membrane were restored. ALA treatment resulted in a significant decrease in autophagy, whose factor may be used against MDS (Camiolo et al., 2019). BM-MSCs showed improved proliferation activity in MDS patients. CDKN2A shows potential as a therapeutic target in regulating the $\mathrm{BM}$ microenvironment because early senescence is reversible via de-induction of CDKN2A (Choi et al., 2020).

Menatetrenone treatment of BM-MSCs enhanced CD34+ cell generation in cocultures by accelerating the cell cycle. MDS-derived cells underwent apoptosis when co-cultured with BM-MSCs, an effect that was enhanced by menatetrenone. These findings indicated that pharmacological treatment with menatetrenone bestows a unique hematopoiesis-supportive capability on BM-MSCs, which may contribute to clinical improvement of cytopenia in MDS (Fujishiro et al., 2020).

Upon exposure to TGF $\beta 1$, healthy MSCs developed functional deficits and adopted a phenotype similar to what was observed in patient-derived stromal cells. These suppressive effects of TGF $\beta 1$ on stromal cell functionality were abrogated by SD-208, an established inhibitor of TGF $\beta$ receptor signaling. Blockade of TGF $\beta$ signaling by SD-208 also restored the osteogenic differentiation capacity of patient-derived stromal cells (Geyh et al., 2018).

All such changes in MDS-MSCs may provide potential targets for the treatment of MDS.

\section{CONCLUSION}

In brief, an increasing number of studies have shown that MDS is a group of heterogeneous diseases caused by abnormalities in both hematopoietic cells and the microenvironment. Tumor hematopoietic cells alter the function of the hematopoietic cell microenvironment via direct contact and secretion of cytokines, causing it to be conducive to the growth of tumor cells. A change in the number and function of MSCs leads to a decline in the supporting function of normal hematopoietic cells, by providing a protective microenvironment for tumor cells, which is conducive to the proliferation of tumor cells. This confers proliferation advantages to tumor cells and reduces tumor cell sensitivity to chemotherapeutic agents and drugs. Gender may be an important reason for the widespread resistance to the disease. If large-sample clinical trials indicate that changes in the number and function of MSCs may be used as potential biomarkers for predicting patient response to treatment or recurrence following therapy, then the changes in MSCs at the time of diagnosis and during treatment will contribute to the formation of personalized therapies.

\section{AUTHOR CONTRIBUTIONS}

$\mathrm{XC}$ and NL searched the literature and drafted part of the manuscript. XD designed the entire study and revised the manuscript. JW revised the manuscript. All authors contributed to the article and approved the submitted version.

\section{FUNDING}

This work was supported by the Nature Science Foundation of China (Nos. 81900132, 82070176, and 82070128), Guangdong Basic and Applied Basic Research Foundation (No. 2019A1515010094), and the Project of Administration of Traditional Chinese Medicine of Guangdong Province (20191008).

\section{ACKNOWLEDGMENTS}

The authors thank the central laboratory of Guangdong Provincial People's Hospital (Guangdong Academy of Medical Sciences). 


\section{REFERENCES}

Abbas, S., Kumar, S., Srivastava, V. M., Therese, M. M., Nair, S. C., Abraham, A., et al. (2019). Heterogeneity of mesenchymal stromal cells in myelodysplastic syndrome-with multilineage dysplasia (MDS-MLD). Indian J. Hematol. Blood Transfus. 35, 223-232. doi: 10.1007/s12288-018-1062-6

Adams, G. B., and Scadden, D. T. (2006). The hematopoietic stem cell in its place, Nat. Immunol. 7, 333-337. doi: 10.1038/ni1331

Alessandrino, E. P., Della Porta, M. G., Bacigalupo, A., Van Lint, M. T., Falda, M., Onida, F., et al. (2008). WHO classification and WPSS predict posttransplantation outcome in patients with myelodysplastic syndrome: a study from the Gruppo Italiano Trapianto di Midollo Osseo (GITMO). Blood 112, 895-902. doi: 10.1182/blood-2008-03-143735

Balderman, S. R., Li, A. J., Hoffman, C. M., Frisch, B. J., Goodman, A. N., Lamere, M. W., et al. (2016). Targeting of the bone marrow microenvironment improves outcome in a murine model of myelodysplastic syndrome. Blood 127:616. doi: 10.1182/blood-2015-06-653113

Barcellos-Hoff, M. H., Park, C., and Wright, E. G. (2005). Radiation and the microenvironment-tumorigenesis and therapy. Nat. Rev. Cancer 5, 867-875. doi: $10.1038 / \mathrm{nrc1} 735$

Boada, M., Echarte, L., Guillermo, C., Diaz, L., Touriño, C., and Grille, S. (2020). 5-Azacytidine restores interleukin 6-increased production in mesenchymal stromal cells from myelodysplastic patients. Hematol. Transfus. Cell Ther. 12, 2531-1387. doi: 10.1016/j.htct.2019.12.002

Bonab, M. M., Alimoghaddam, K., Talebian, F., Ghaffari, S. H., Ghavamzadeh, A., and Nikbin, B. (2006). Aging of mesenchymal stem cell in vitro. BMC Cell Biol. 7:14. doi: $10.1186 / 1471-2121-7-14$

Camiolo, G., Tibullo, D., Giallongo, C., Romano, A., Parrinello, N. L., Musumeci, G., et al. (2019). $\alpha$-Lipoic acid reduces iron-induced toxicity and oxidative stress in a model of iron overload. Int. J. Mol. Sci. 20:609. doi: 10.3390/ijms20030609

Casadevall, N., Durieux, P., Dubois, S., Hemery, F., Lepage, E., Quarré, M. C., et al. (2004). Health, economic, and quality-of-life effects of erythropoietin and granulocyte colony-stimulating factor for the treatment of myelodysplastic syndromes: a randomized, controlled trial. Blood 104, 321-327. doi: 10.1182/blood-2003-07-2252

Cheson, B. D., Bennett, J. M., Kantarjian, H., Pinto, A., Schiffer, C. A., Nimer, S. D., et al. (2000). Report of an international working group to standardize response criteria for myelodysplastic syndromes. Blood 96, 3671-3674. doi: 10.1182/blood.V96.12.3671

Cheson, B. D., Greenberg, P. L., Bennett, J. M., Lowenberg, B., Wijermans, P. W., Nimer, S. D., et al. (2006). Clinical application and proposal for modification of the International Working Group (IWG) response criteria in myelodysplasia. Blood 108, 419-425. doi: 10.1182/blood-2005-10-4149

Choi, H., Kim, Y., Kang, D., Kwon, A., Kim, J., Min Kim, J., et al. (2020). Common and different alterations of bone marrow mesenchymal stromal cells in myelodysplastic syndrome and multiple myeloma. Cell Prolif. 53:e12819. doi: $10.1111 /$ cpr.12819

Colmone, A., Amorim, M., Pontier, A. L., Wang, S., Jablonski, E., and Sipkins, D. A. (2008). Leukemic cells create bone marrow niches that disrupt the behavior of normal hematopoietic progenitor cells. Science 322, 1861-1865. doi: $10.1126 /$ science. 1164390

Dührsen, U., and Metcalf, D. (1990). Effects of irradiation of recipient mice on the behavior and leukemogenic potential of factor-dependent hematopoietic cell lines. Blood 75, 190-197. doi: 10.1182/blood.V75.1. 190.190

Falconi, G., Fabiani, E., Fianchi, L., Criscuolo, M., Raffaelli, C. S., Bellesi, S., et al. (2016). Impairment of PI3K/AKT and WNT/ $\beta$-catenin pathways in bone marrow mesenchymal stem cells isolated from patients with myelodysplastic syndromes. Exp. Hematol. 44, 75-83.e71-74. doi: 10.1016/j.exphem.2015.10.005

Fei, C., Zhao, Y., Guo, J., Gu, S., Li, X., and Chang, C. (2014). Senescence of bone marrow mesenchymal stromal cells is accompanied by activation of p53/p21 pathway in myelodysplastic syndromes. Eur. J. Haematol. 93, 476-486. doi: 10.1111/ejh.12385

Fei, C. M., Guo, J., Zhao, Y. S., Zhao, S. D., Zhen, Q. Q., Shi, L., et al. (2018). Clinical significance of hyaluronan levels and its pro-osteogenic effect on mesenchymal stromal cells in myelodysplastic syndromes. J. Transl. Med. 16:234. doi: 10.1186/s12967-018-1614-4
Ferrer, R. A., Wobus, M., List, C., Wehner, R., Schönefeldt, C., Brocard, B., et al. (2013). Mesenchymal stromal cells from patients with myelodyplastic syndrome display distinct functional alterations that are modulated by lenalidomide. Haematologica 98, 1677-1685. doi: 10.3324/haematol.2013.083972

Flynn, C. M., and Kaufman, D. S. (2007). Donor cell leukemia: insight into cancer stem cells and the stem cell niche. Blood 109, 2688-2692. doi: 10.1182/blood-2006-07-021980

Fujishiro, A., Iwasa, M., Fujii, S., Maekawa, T., Andoh, A., Tohyama, K., et al. (2020). Menatetrenone facilitates hematopoietic cell generation in a manner that is dependent on human bone marrow mesenchymal stromal/stem cells. Int. J. Hematol. 112, 316-330. doi: 10.1007/s12185-020-02916-8

Geyh, S., Oz, S., Cadeddu, R. P., Fröbel, J., Brückner, B., Kündgen, A., et al. (2013). Insufficient stromal support in MDS results from molecular and functional deficits of mesenchymal stromal cells. Leukemia 27, 1841-1851. doi: 10.1038/leu.2013.193

Geyh, S., Rodríguez-Paredes, M., Jäger, P., Koch, A., Bormann, F., Gutekunst, J., et al. (2018). Transforming growth factor $\beta 1$-mediated functional inhibition of mesenchymal stromal cells in myelodysplastic syndromes and acute myeloid leukemia. Haematologica 103, 1462-1471. doi: 10.3324/haematol.2017.186734

Glenthøj, A., Ørskov, A. D., Hansen, J. W., Hadrup, S. R., O'Connell, C., and Grønbæk, K. (2016). Immune mechanisms in myelodysplastic syndrome. Int. J. Mol. Sci. 17:944. doi: 10.3390/ijms17060944

Hellström-Lindberg, E. (1995). Efficacy of erythropoietin in the myelodysplastic syndromes: a meta-analysis of 205 patients from 17 studies. Br. J. Haematol. 89, 67-71. doi: 10.1111/j.1365-2141.1995.tb08909.x

Hellström-Lindberg, E., Negrin, R., Stein, R., Krantz, S., Lindberg, G., Vardiman, J., et al. (1997). Erythroid response to treatment with GCSF plus erythropoietin for the anaemia of patients with myelodysplastic syndromes: proposal for a predictive model. Br. J. Haematol. 99, 344-351. doi: 10.1046/j.1365-2141.1997.4013211.x

Hicks, L. K., Bering, H., Carson, K. R., Kleinerman, J., Kukreti, V., $\mathrm{Ma}$, A., et al. (2013). The ASH choosing Wisely® campaign: five hematologic tests and treatments to question. Blood 122, 3879-3883. doi: 10.1182/blood-2013-07-518423

Isern, J., García-García, A., Martín, A. M., Arranz, L., Martín-Pérez, D., Torroja, C., et al. (2014). The neural crest is a source of mesenchymal stem cells with specialized hematopoietic stem cell niche function. eLife 3:e03696. doi: 10.7554/eLife.03696.021

Iwata, M., Pillai, M., Ramakrishnan, A., Hackman, R. C., Deeg, H. J., Opdenakker, G., et al. (2007). Reduced expression of inducible gelatinase B/matrix metalloproteinase- 9 in monocytes from patients with myelodysplastic syndrome: correlation of inducible levels with the percentage of cytogenetically marked cells and with marrow cellularity. Blood 109, 85-92. doi: 10.1182/blood-2006-05-020289

Jabbour, E., Short, N. J., Montalban-Bravo, G., Huang, X., Bueso-Ramos, C., Qiao, W., et al. (2017). Randomized phase 2 study of low-dose decitabine vs lowdose azacitidine in lower-risk MDS and MDS/MPN. Blood 130, 1514-1522. doi: 10.1182/blood-2017-06-788497

Kfoury, Y., and Scadden, D. T. (2015). Mesenchymal cell contributions to the stem cell niche. Cell Stem Cell 16, 239-253. doi: 10.1016/j.stem.2015.02.019

Komada, Y., Yamane, T., Kadota, D., Isono, K., Takakura, N., Hayashi, S., et al. (2012). Origins and properties of dental, thymic, and bone marrow mesenchymal cells and their stem cells. PLoS ONE 7:e46436. doi: 10.1371 /journal.pone. 0046436

Li, A. J., and Calvi, L. M. (2017). The microenvironment in myelodysplastic syndromes: niche-mediated disease initiation and progression. Exp. Hematol. 55, 3-18. doi: 10.1016/j.exphem.2017.08.003

Li, Z., and Li, L. (2006). Understanding hematopoietic stemcell microenvironments. Trends Biochem. Sci. 31, 589-595. doi: 10.1016/j.tibs.2006.08.001

Lin, Y.-W., Slape, C., Zhang, Z., and Aplan, P. D. (2005). NUP98-HOXD13 transgenic mice develop a highly penetrant, severe myelodysplastic syndrome that progresses to acute leukemia. Blood 106, 287-295. doi: 10.1182/blood-2004-12-4794

Liu, Q., Zhu, H., Dong, J., Li, H., and Zhang, H. (2015). Defective proliferative potential of MSCs from pediatric myelodysplastic syndrome patients is associated with cell senescence. Int. J. Clin. Exp. Pathol. 8, 13059-13066. 
Maes, C., Kobayashi, T., Selig, M. K., Torrekens, S., Roth, S. I., Mackem, S., et al. (2010). Osteoblast precursors, but not mature osteoblasts, move into developing and fractured bones along with invading blood vessels. Dev. Cell 19, 329-344. doi: 10.1016/j.devcel.2010.07.010

Mellibovsky, L., Diez, A., Serrano, S., Aubia, J., Pérez-Vila, E., Mariñoso, M. L., et al. (1996). Bone remodeling alterations in myelodysplastic syndrome. Bone 19, 401-405. doi: 10.1016/S8756-3282(96)00210-4

Méndez-Ferrer, S., Michurina, T. V., Ferraro, F., Mazloom, A. R., Macarthur, B. D., Lira, S. A., et al. (2010). Mesenchymal and haematopoietic stem cells form a unique bone marrow niche. Nature 466, 829-834. doi: 10.1038/nature09262

Mizoguchi, T., Pinho, S., Ahmed, J., Kunisaki, Y., Hanoun, M., Mendelson, A., et al. (2014). Osterix marks distinct waves of primitive and definitive stromal progenitors during bone marrow development. Dev. Cell 29, 340-349. doi: 10.1016/j.devcel.2014.03.013

Moore, K. A., and Lemischka, I. R. (2006). Stem cells and their niches. Science 311, 1880-1885. doi: 10.1126/science. 1110542

Negrin, R. S., Stein, R., Doherty, K., Cornwell, J., Vardiman, J., Krantz, S., et al. (1996). Maintenance treatment of the anemia of myelodysplastic syndromes with recombinant human granulocyte colony-stimulating factor and erythropoietin: evidence for in vivo synergy. Blood 87, 4076-4081. doi: 10.1182/blood.V87.10.4076.bloodjournal87104076

Oliva, E. N., Latagliata, R., Laganà, C., Breccia, M., Galimberti, S., Morabito, F., et al. (2013). Lenalidomide in International Prognostic Scoring System low and intermediate- 1 risk myelodysplastic syndromes with del $(5 q)$ : an Italian phase II trial of health-related quality of life, safety and efficacy. Leuk. Lymphoma. 54, 2458-2465. doi: 10.3109/10428194.2013.778406

Ono, N., Ono, W., Mizoguchi, T., Nagasawa, T., Frenette, P. S., and Kronenberg, H. M. (2014a). Vasculature-associated cells expressing nestin in developing bones encompass early cells in the osteoblast and endothelial lineage. Dev. Cell 29, 330-339. doi: 10.1016/j.devcel.2014.03.014

Ono, N., Ono, W., Nagasawa, T., and Kronenberg, H. M. (2014b). A subset of chondrogenic cells provides early mesenchymal progenitors in growing bones. Nat. Cell Biol. 16, 1157-1167. doi: 10.1038/ncb3067

Park, D., Spencer, J. A., Koh, B. I., Kobayashi, T., Fujisaki, J., Clemens, T. L., et al. (2012). Endogenous bone marrow MSCs are dynamic, fate-restricted participants in bone maintenance and regeneration. Cell Stem Cell 10, 259-272. doi: 10.1016/j.stem.2012.02.003

Pavlaki, K., Pontikoglou, C. G., Demetriadou, A., Batsali, A. K., Damianaki, A., Simantirakis, E., et al. (2014). Impaired proliferative potential of bone marrow mesenchymal stromal cells in patients with myelodysplastic syndromes is associated with abnormal WNT signaling pathway. Stem Cells Dev 23, 1568-1581. doi: 10.1089/scd.2013.0283

Pfeilstöcker, M., Tuechler, H., Sanz, G., Schanz, J., Garcia-Manero, G., Sol,é, F., et al. (2016). Time-dependent changes in mortality and transformation risk in MDS. Blood 128, 902-910. doi: 10.1182/blood-2016-02-7 00054

Pleyer, L., Valent, P., and Greil, R. (2016). Mesenchymal stem and progenitor cells in normal and dysplastic hematopoiesis-masters of survival and clonality? Int. J. Mol. Sci. 17:1009. doi: 10.3390/ijms17071009

Raaijmakers, M. H. (2012). Myelodysplastic syndromes: revisiting the role of the bone marrow microenvironment in disease pathogenesis. Int. J. Hematol. 95, 17-25. doi: 10.1007/s12185-011-1001-x

Revicki, D. A., Brandenburg, N. A., Muus, P., Yu, R., Knight, R., and Fenaux, P. (2013). Health-related quality of life outcomes of lenalidomide in transfusiondependent patients with Low- or Intermediate-1-risk myelodysplastic syndromes with a chromosome $5 \mathrm{q}$ deletion: results from a randomized clinical trial. Leuk. Res. 37, 259-265. doi: 10.1016/j.leukres.2012.11.017

Sacchetti, B., Funari, A., Michienzi, S., Di Cesare, S., Piersanti, S., Saggio, I., et al. (2007). Self-renewing osteoprogenitors in bone marrow sinusoids can organize a hematopoietic microenvironment. Cell 131, 324-336. doi: 10.1016/j.cell.2007.08.025

Schepers, K., Pietras, E. M., Reynaud, D., Flach, J., Binnewies, M., Garg, T., et al. (2013). Myeloproliferative neoplasia remodels the endosteal bone marrow niche into a self-reinforcing leukemic niche. Cell Stem Cell 13, 285-299. doi: 10.1016/j.stem.2013.06.009

Shi, L., Zhao, Y., Fei, C., Guo, J., Jia, Y., Wu, D., et al. (2019). Cellular senescence induced by S100A9 in mesenchymal stromal cells through NLRP3 inflammasome activation. Aging 11, 9626-9642. doi: 10.18632/aging.102409

Silverman, L. R., Fenaux, P., Mufti, G. J., Santini, V., Hellström-Lindberg, E., Gattermann, N., et al. (2011). Continued azacitidine therapy beyond time of first response improves quality of response in patients with higher-risk myelodysplastic syndromes. Cancer 117, 2697-2702. doi: 10.1002/cncr.25774

Spiriti, M. A., Latagliata, R., Niscola, P., Cortelezzi, A., Francesconi, M., Ferrari, D., et al. (2005). Impact of a new dosing regimen of epoetin alfa on quality of life and anemia in patients with low-risk myelodysplastic syndrome. Ann. Hematol. 84, 167-176. doi: 10.1007/s00277-004-0961-9

Stenderup, K., Justesen, J., Clausen, C., and Kassem, M. (2003). Aging is associated with decreased maximal life span and accelerated senescence of bone marrow stromal cells. Bone 33, 919-926. doi: 10.1016/j.bone.2003.07.005

Takashima, Y., Era, T., Nakao, K., Kondo, S., Kasuga, M., Smith, A. G., et al. (2007). Neuroepithelial cells supply an initial transient wave of MSC differentiation. Cell 129, 1377-1388. doi: 10.1016/j.cell.2007.04.028

Verstovsek, S., Estey, E., Manshouri, T., Giles, F. J., Cortes, J., Beran, M., et al. (2002). Clinical relevance of vascular endothelial growth factor receptors 1 and 2 in acute myeloid leukaemia and myelodysplastic syndrome. Br. J. Haematol. 118, 151-156. doi: 10.1046/j.1365-2141.2002.03551.x

Weidner, H., Rauner, M., Trautmann, F., Schmitt, J., Balaian, E., Mies, A., et al. (2017). Myelodysplastic syndromes and bone loss in mice and men. Leukemia 31:1003. doi: 10.1038/leu.2017.7

Wright, E. G. (2005). Ionizing radiation and leukaemia: more questions than answers. Hematol. Oncol. 23, 119-126. doi: 10.1002/hon.763

Zhao, Y., Wu, D., Fei, C., Guo, J., Gu, S., Zhu, Y., et al. (2015). Downregulation of Dicer1 promotes cellular senescence and decreases the differentiation and stem cell-supporting capacities of mesenchymal stromal cells in patients with myelodysplastic syndrome. Haematologica 100, 194-204. doi: 10.3324/haematol.2014.109769

Zhao, Z.-G., Xu, W., Yu, H.-P., Fang, B.-L., Wu, S.-H., Li, F., et al. (2012). Functional characteristics of mesenchymal stem cells derived from bone marrow of patients with myelodysplastic syndromes. Cancer Lett. 317, 136-143. doi: 10.1016/j.canlet.2011.08.030

Zheng, Q., Zhao, Y., Guo, J., Zhao, S., Fei, C., Xiao, C., et al. (2018). Iron overload promotes mitochondrial fragmentation in mesenchymal stromal cells from myelodysplastic syndrome patients through activation of the AMPK/MFF/Drp1 pathway. Cell Death Dis. 9:515. doi: 10.1038/s41419-018-0552-7

Zhou, B. O., Yue, R., Murphy, M. M., Peyer, J. G., and Morrison, S. J. (2014). Leptin-receptor-expressing mesenchymal stromal cells represent the main source of bone formed by adult bone marrow. Cell Stem Cell 15, 154-168. doi: 10.1016/j.stem.2014. 06.008

Zhou, S., Greenberger, J. S., Epperly, M. W., Goff, J. P., Adler, C., Leboff, M. S., et al. (2008). Age-related intrinsic changes in human bonemarrow-derived mesenchymal stem cells and their differentiation to osteoblasts. Aging Cell 7, 335-343. doi: 10.1111/j.1474-9726.2008. 00377.x

Conflict of Interest: The authors declare that the research was conducted in the absence of any commercial or financial relationships that could be construed as a potential conflict of interest.

Copyright (C) 2021 Chen, Li, Weng and Du. This is an open-access article distributed under the terms of the Creative Commons Attribution License (CC BY). The use, distribution or reproduction in other forums is permitted, provided the original author(s) and the copyright owner(s) are credited and that the original publication in this journal is cited, in accordance with accepted academic practice. No use, distribution or reproduction is permitted which does not comply with these terms. 